\title{
Human Detection and Discrimination of Tactile Repeatability, Mechanical Backlash, and Temporal Delay in a Combined Tactile-Kinesthetic Haptic Display System
}

\author{
Andrew J. Doxon, Student Member, IEEE, David E. Johnson, \\ Hong Z. Tan, Senior Member, IEEE, and William R. Provancher, Member, IEEE
}

\begin{abstract}
Many of the devices used in haptics research are over-engineered for the task and are designed with capabilities that go far beyond human perception levels. Designing devices that more closely match the limits of human perception will make them smaller, less expensive, and more useful. However, many device-centric perception thresholds have yet to be evaluated. To this end, three experiments were conducted, using one degree-of-freedom contact location feedback device in combination with a kinesthetic display, to provide a more explicit set of specifications for similar tactile-kinesthetic haptic devices. The first of these experiments evaluated the ability of humans to repeatedly localize tactile cues across the fingerpad. Subjects could localize cues to within $1.3 \mathrm{~mm}$ and showed bias toward the center of the fingerpad. The second experiment evaluated the minimum perceptible difference of backlash at the tactile element. Subjects were able to discriminate device backlash in excess of $0.46 \mathrm{~mm}$ on low-curvature models and $0.93 \mathrm{~mm}$ on high-curvature models. The last experiment evaluated the minimum perceptible difference of system delay between user action and device reaction. Subjects were able to discriminate delays in excess of $61 \mathrm{~ms}$. The results from these studies can serve as the maximum (i.e., most demanding) device specifications for most tactile-kinesthetic haptic systems.
\end{abstract}

Index Terms-Evaluation of haptic devices, haptic device design, haptic 1/O, psychophysics

\section{INTRODUCTION}

M ANY haptic devices used in research applications are overengineered for their given task. While this provides additional benefits in some fields, it serves as a detriment in the field of haptics. Once a device's performance has exceeded the limits of human perception, any additional precision provides no further benefit, unless directly trying to measure human perception capabilities. Understanding these perceptual limits can provide a more explicit set of specifications for haptic device designs. By closely matching these specifications, haptic devices can become smaller, less expensive, and more useful, expanding their presence as both research and commercial products.

To begin addressing the issue, this paper presents three experiments evaluating perceptual thresholds relating to tactile device design. Each of the following experiments are performed with a tactile device known as a contact location

- A.J. Doxon and W.R. Provancher are with the Department of Mechanical Engineering, College of Engineering, University of Utah, Salt Lake City, UT 84112. E-mail: adoxon@gmail.com, wil@mech.utah.edu.

- D.E. Johnson is with the School of Computing, College of Engineering, University of Utah, Salt Lake City, UT 84112. E-mail:dejohnso@cs.utah.edu.

- H.Z. Tan is with the School of Electrical and Computer Engineering, College of Engineering, Purdue University, EE Building, 465 Northwestern Avenue, West Lafayette, IN 47907. E-mail: hongtan@purdue.edu.

Manuscript received 6 May 2013; revised 7 Aug. 2013; accepted 27 Aug. 2013; published online 26 Sept. 2013.

Recommended for acceptance by G. Baud-Bovy.

For information on obtaining reprints of this article, please send e-mail to: toh@computer.org, and reference IEEECS Log Number TH-2013-05-0055.

Digital Object Identifier no. 10.1109/ToH.2013.50. display (CLD), described in detail in Section 3, and the results can serve as the maximum (i.e., most demanding) device specifications for similar tactile-kinesthetic haptic systems. That is, our results apply to systems that provide tactile feedback where the user is experiencing tactile feedback as a function of their limb motions. The first of these three experiments identifies the resolution with which tactile cues can be repeatedly localized on the distal fingerpad. We ask participants to match a touched point on the fingertip by actively adjusting the location of a tactor on the same fingertip. The measured error of this repeated localization procedure provides the maximum positioning error allowed by the tactor in order for two placements of the tactor on the fingertip to be perceived as being at the same position. The second experiment evaluates the minimum perceivable difference in device backlash when positioning a tactile element. Backlash provides both physical cues, through positioning error during motion, and temporal cues, through a delay in the onset of tactor motion after finger motion. As backlash detection is heavily dependent on tactor positioning, the experiment was performed on both a low-curvature and a high-curvature surface. The results indicate the level of backlash that can be present in a device before it becomes detectable. The third experiment measures the minimum perceivable difference in system delay between user action and device motion. As with backlash, system delay can manifest in both physical and temporal cues. However, the magnitude of these cues is tied with tactor velocity rather than position and thus is 
often masked by user motion. The detection of system delay was evaluated as a whole and with only the cues provided at the onset of motion. These results provide the maximum amount of system delay that can be present before it becomes noticeable.

The following section provides a brief background concerning the literature most relevant to this research. This is followed by a description of the CLD device and an overview of the experiments performed. Each of the three experiments is then presented in turn, with results and discussion. Finally, results from all experiments are summarized and future work is discussed.

\section{BACKGROUND}

\subsection{Human Sensing Thresholds}

A substantial amount of work has been published regarding the haptic sensing abilities of humans. Biggs and Srinivasan [1] and Hale and Stanney [2] both provide a compilation of some prior work, tabulating their results into an easy-to-use.

When designing tactile devices, it is important to understand how users judge spatial-tactile information provided to their fingertips. Human ability to localize tactile cues on the fingertip varies as a function of the cue type being given. Textures and microbumps, some of the smallest shapes that can be tactilely perceived, are detected through vibrations and skin stretch. Gleeson et al. [3] demonstrated that subjects could detect the direction of skin displacements of $50 \mu \mathrm{m}$ in the cardinal directions. Loomis and Collins [4] also demonstrated that these skin stretch cues could be detected with a much finer resolution than single-point localization. Loomis also evaluated fingerpad localization with respect to successive single-point tactile cues. Subjects were asked to identify whether the subsequent cue was provided to the right or left of the prior cue. This experiment showed subjects were able to localize cue positions and displacements as fine as $0.17 \mathrm{~mm}$ [5]. This localization is different from the two-point limen, which is the minimum separation distance at which two simultaneous cues can each be sensed individually. Boven and Johnson [6] report the two-point limen at multiple locations on the body. They report the two-point limen at the fingertip to be around $0.94 \mathrm{~mm}$.

An arguably more important threshold to keep in mind when designing tactile devices is that of temporal delay. Many publications have shown that long delays, such as those caused when communicating across networks, can cause significant performance decreases in positioning and manipulation tasks. The vast majority of these studies have investigated the effects of audio and visual delays on performance and perception. Other studies have shown the effects of network delays on kinesthetic haptic interaction [7]. However, relatively little research has been performed with respect to the effect of time delays on user performance in tactile-kinesthetic haptic systems. Of these three domains (audio, visual, and haptic), delays in audio feedback are the most perceptible. Adelstein et al. [8] showed audio delay with respect to a visual image became detectable at around $20 \mathrm{~ms}$. Mania et al. [9] found visual delays with respect to head motion are usually detected around $40 \mathrm{~ms}$ but could be detected as low as $30 \mathrm{~ms}$. Jay and Hubbold [10] demonstrated visual delays above $69 \mathrm{~ms}$ significantly hindered user performance in a Fitts-type task. In a similar Fitts-type task, Jay and Hubbold also showed that providing delay in haptic feedback is less disruptive than in visual feedback. In this task, the target area was kinesthetically rendered as a solid plane, giving the sensation of striking a solid surface. They found haptic delays in excess of $187 \mathrm{~ms}$ to cause a statistically significant performance decrease [10].

While not directly related to a single perception threshold, device backlash should also be considered when designing haptic devices. While most authors agree there should be little-to-no backlash in haptic systems, they rarely report their device's backlash or what an ideal level of backlash should be. Backlash detection can be viewed as a combination of other perception thresholds. Backlash can be sensed as either a small displacement error or as a time delay in device motion based on user velocity. In either case, the resulting minimum detectable backlash is small (likely $100 \mathrm{~s}$ of micrometers or $10 \mathrm{~s}$ of milliseconds in scale).

In addition to accounting for tactile perception thresholds, haptic devices should have a bandwidth in excess of their users' and adequate to faithfully render a given virtual environment. Humans are generally estimated to have a maximum bandwidth between 5 and $10 \mathrm{~Hz}$ [11]. While user velocity is slower during exploration, tactile device positioning is also affected by changes in surface contours. These relative changes can easily create high-frequency tactile cues in excess of $10 \mathrm{~Hz}$. However, very little research has investigated finger velocities during tactile exploration. Generally, tactile devices are designed with high bandwidth to overcome this problem. Frisoli et al. [12] and Lederman and Klatzky [13], [14] discuss the motions and methods of tactile exploration. These motions and methods provide a basic estimate of the relative finger velocities under different exploration conditions.

\subsection{Design Guidelines for Tactile Displays}

Drewing et al. [16] and Webster et al. [15] discuss some basic guidelines for designing tactile displays. In particular, they place the greatest importance on matching human perception thresholds and miniaturization when designing devices for use in combination with tactile and kinesthetic feedback. Webster et al. also point out that the device's tactor velocities should be capable of exceeding maximum finger exploration velocities. They estimate a safe upper bound for tactor velocities of $30-40 \mathrm{~cm} / \mathrm{s}$ [15]. The results of both of these studies were applied to their next generation of slip displays.

In related work, Salisbury et al. [17] evaluate the performance of commercially available haptic devices when rendering textures. Their results provide device design guidelines to ensure proper rendering of the vibratory components of textures.

Other publications suggest guidelines for the design of vibrotactile feedback [18], [19] and pin arrays [20], but a number of design parameters for other tactile systems must be gathered from the related psychophysics literature. Little other work discusses design guidelines for building combined tactile and kinesthetic devices. 

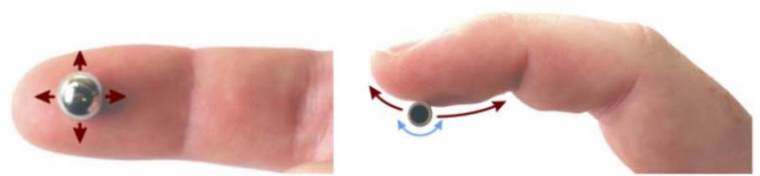

Fig. 1. Concept for contact location feedback. The (left) two-dimensional or (right) one-dimensional center of contact is represented with a single tactile element. The current contact location display is only capable of displaying one-dimensional contacts along the length of the finger (see Fig. 2).

The results of the present study can be viewed as the most demanding specifications for combined tactile-kinesthetic haptic-feedback systems, including those that display slip (e.g., [21], [22], [15], [16]), tactile pin array (e.g., [22], [23]), contact orientation (e.g., [24], [25], [26]), and contact location [27].

\section{Experimental Apparatus}

The basic concept of contact location is presented in Fig. 1. Rather than providing all possible tactile information to the user, only the center of contact is rendered through a small tactor. In the current device design, the tactor is only capable of motions in the proximal-distal directions. The contact location display device is mounted to a SensAble Phantom Premium 1.5 through a 3 degrees-of-freedom gimbal to allow full motion of the finger. The Phantom provides the kinesthetic force feedback of the system while the contact location display provides tactile feedback.

The device utilizes a 1-cm diameter delrin roller as the tactile contact element (tactor). This ensures that only the contact position is provided and no skin stretch is experienced when the contactor is moved along the finger. The position of the roller is controlled via two sheathed push-pull wires attached to a linear actuator mounted on the user's forearm. An open-bottomed thimble is used to securely attach the device to the user's finger. Different sized thimbles can be interchanged onto the CLD to accommodate a wide range of finger sizes. The thimble also provides the anchor points for the push-pull wire sheaths, ensuring the push-pull wires are never in contact with the skin. The roller is held continuously in contact with the fingerpad by two small springs attached to the thimble. Forces are applied to the finger directly through the openbottomed thimble.

The linear actuator is located on the user's forearm to minimize device inertia at the fingertip and prevent any actuator vibrations from being transmitted to the user's fingertip. While some low-magnitude actuator vibrations may be detected by the forearm, the influence of these vibrations is effectively eliminated by the relatively lower sensitivity of the forearm and the user's attention being focused at the fingertip. The user's forearm is supported by a rolling arm rest to allow comfortable positioning of the finger. The linear actuator utilizes a Faulhaber DC Micromotor (1724-024S) with 3.71:1 gearbox and a 3.175-mm pitch leadscrew with an antibacklash nut to provide approximately $2 \mathrm{~cm}$ of linear motion. The device has approximately $0.4 \mu \mathrm{m}$ of resolution and a bandwidth in excess of $5 \mathrm{~Hz}$. Device backlash at the tactor was characterized to be $0.23 \mathrm{~mm}$ throughout its workspace. This backlash is primarily caused

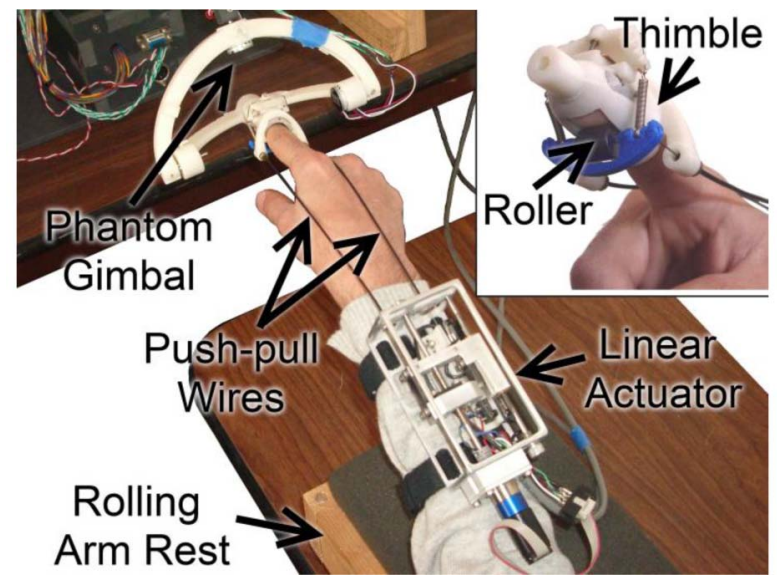

Fig. 2. CLD attached to a Phantom robot. The user's elbow is supported by a rolling armrest. The user's finger is secured to the CLD via an openbottomed thimble.

by deformations in the push-pull wire sheaths due to friction between the push-pull wires and sheaths. The current device, attached to a Phantom through a 3 degrees-of-freedom gimbal, can be seen in Fig. 2. A close up view of the fingertip portion of the device is also shown in Fig. 2.

The device's motor is driven by an AMC 12A8 PWM current amplifier controlled using a Sensoray 626 PCI control card. The device's position is controlled through a PID controller run at $1 \mathrm{kHz}$. This controller was programmed in $\mathrm{C}++$ and executed in a Windows 7 environment using Windows multimedia timers. Further details about the design and control of this device are related to the earlier version of the device found in [27].

\section{General Methods}

Three separate experiments were conducted to evaluate perceptual thresholds relating to tactile device design. The results from these experiments can be applied to most tactile-kinesthetic haptic systems. The first of these three experiments identifies the resolution with which users are able to repeatedly localize tactile cues at a given location on their fingerpad. This directly identifies the maximum positioning error a tactile device can have before the error becomes noticeable. The second experiment evaluates the minimum perceivable amount of backlash when positioning a tactile element on the user's fingerpad. Most haptic devices are designed to contain virtually no backlash. Designing closer to the perceptual limit will help relax design tolerances and reduce system costs. Lastly, the third experiment measures the minimum perceivable system delay between user action and device motion. For haptic devices to feel responsive, the whole system delay must be less than the measured value.

During each experiment, the velocities of both the tactor and finger were recorded. These velocities help to identify common interaction speeds when exploring virtual environments. Devices unable to react at these velocities will feel sluggish and unresponsive.

The above experiments are conducted using the contact location display, described in Section 3, whose performance exceeds the expected human perceptual limits in all the 


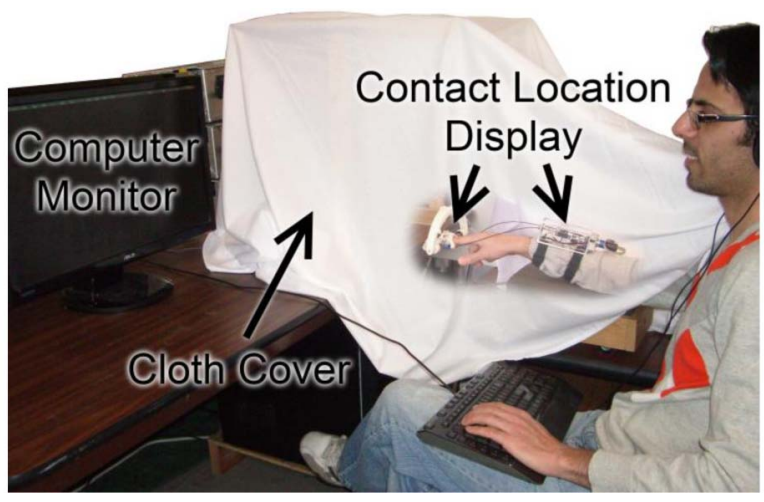

Fig. 3. Experiment setup (cover made transparent for clarity).

above cases. One of our goals resulting from these experiments is to miniaturize the design of this tactile display.

Each experiment was performed by the same group of 20 participants ( 3 female, 3 left handed). Participant ages ranged between 20 and 40 . Half of the participants had prior experience using the CLD device.

All three experiments were performed in the same session. A Latin squares reduction was used to determine experiment order to provide balanced testing. Before each experiment, participants underwent a brief training period to familiarize them with the experiment's task and response process. Each experiment took around 15-20 minutes to complete, with all three experiments taking approximately 1-1.5 hours in total, including breaks. Participants took breaks between experiments and sections within each experiment to reduce fatigue effects.

The participant's arm and testing apparatus were obscured by a cloth cover throughout the duration of each experiment. Experiment instructions were provided on the computer monitor, but no other visual feedback was provided. White noise was played on noise-cancelling headphones during testing to eliminate any auditory cues from device motion. Additional audio cues were provided to assist in pacing the experiment and to indicate transitions between stimuli. The experimental setup can be seen in Fig. 3.

Each of the three experiments utilizes the same base environment. This environment consists of a single $95-\mathrm{mm}$ radius cylinder with its axis of symmetry aligned horizontally from right to left. The cylinder model was chosen to provide an object surface with a constant curvature. The fore-aft motion of the participant's finger along the curved surface is natural and comfortable given the kinematics of the CLD as compared to the movement required by a planar surface to achieve the same interaction. The user's virtual finger is represented by a 13-mm sphere, offset such that its surface aligns with the user's fingerpad. Fig. 4 shows a representation of the virtual environment used in the experiments.

\section{Repeated Localization of Tactile Cues}

The first experiment evaluates the resolution with which users are able to repeatedly locate tactile contact on their fingerpad through a position matching task. This directly identifies the maximum positioning error a tactile device

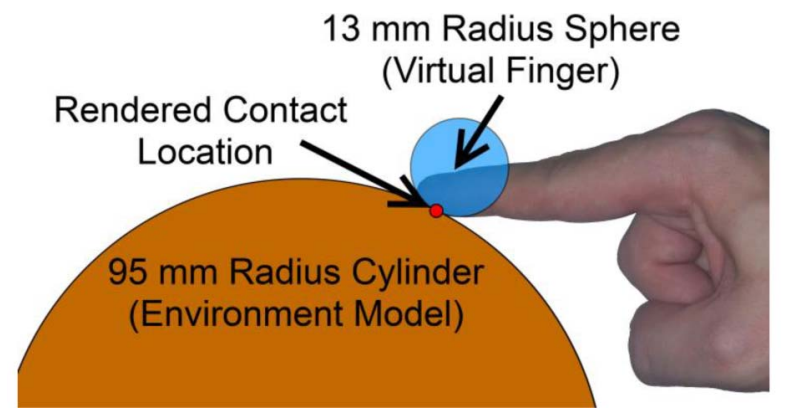

Fig. 4. The virtual environment used in each of the three experiments. This environment was slightly altered in some of the experiments.

can contain before the error becomes noticeable for sequential contacts. Other studies have clearly shown that even extremely small tactor motions can be detected [28]. Thus, device designs should take into account the expected form of tactor motions during use when determining the amount of acceptable positioning error.

\subsection{Methods}

Users were instructed to match successive tactile contact locations through interacting with a cylindrical model. The model's position and radius vary but functionally is the same as the base environment described in Section 4.

\subsubsection{Procedure}

The position matching (repeated localization) task was evaluated at five points along the length of the fingerpad (see Fig. 5). These positions are evenly distributed across the workspace of the CLD. The edges of the workspace were avoided as they provide additional references (perceptual anchors) that would artificially increase people's performance at those locations [29]. The spacing between test locations is approximately $2.8 \mathrm{~mm}$. This is larger than the two point limen which indicates a different set of mechanoreceptors is being tested with each location [6]. Fig. 5 shows the test locations on the fingerpad, with the labeled points corresponding with those in Figs. 7 and 8. The participants' ability to place the contact was evaluated at each of these five locations. Each location was tested 10 times, with the order of the 50 trials randomized for each participant.

Each trial consisted of the following sequence: First, a visual representation of the current tactor position and a target region was shown on the computer monitor (see Fig. 6). The current tactor position is represented by a red sphere. The target region is represented by a green

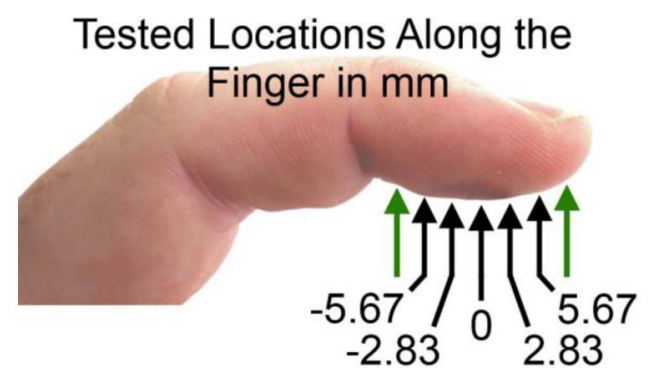

Fig. 5. Five test locations along the length of the fingerpad. Test locations separated by about $2.8 \mathrm{~mm}$. The green arrows denote the edges of the CLD's workspace. 


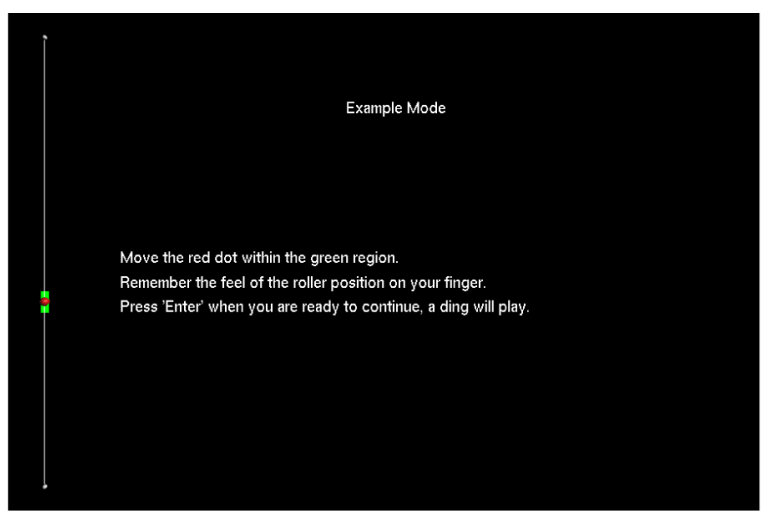

Fig. 6. Graphics and instructions displayed to the participant during the experiment. An indicator of tactor position is shown on the left. The red sphere represents the tactor location. The green rectangle represents the target area to proceed. Instructions are shown in the center of the screen.

rectangle centered about the chosen test location and spans $\pm 0.25 \mathrm{~mm}$. A participant then moves his/her finger such that the tactor position was within the green rectangle. Once there, the participant was instructed to hold their arm stationary and memorize the position of the tactor on their fingerpad. The participant indicated they were ready to proceed by pressing "Enter" on the keyboard. A tone would sound and the visual indicator of position was removed, indicating their current position was recorded. The user would then raise their finger above the surface while the radius and position of the cylinder was altered. A second tone would sound to indicate the participant could lower his/her finger onto the new virtual surface. Once on the new surface, the participant moved his/her finger fore/aft such that the tactor's position on his/her fingerpad matched the previously recorded position, to the best of their ability. The participant finished the trial by pressing "Enter" again to record his/her current "matched" position. The visual indicator of position was displayed again and the next trial would begin.

\subsubsection{Stimuli}

The environment model is a horizontal cylinder similar to the base environment described by Fig. 4 in Section 4 . Between each matching task the position and radius of the

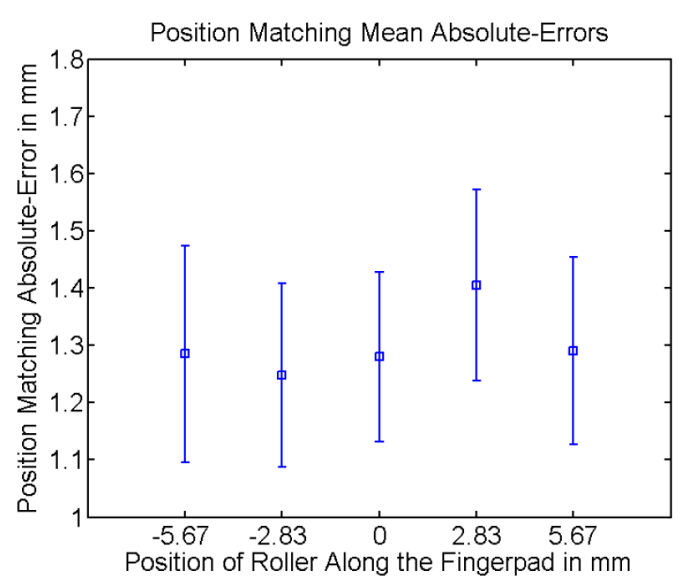

Fig. 7. Mean absolute error and its 95 percent confidence intervals among the five test locations across all participants.

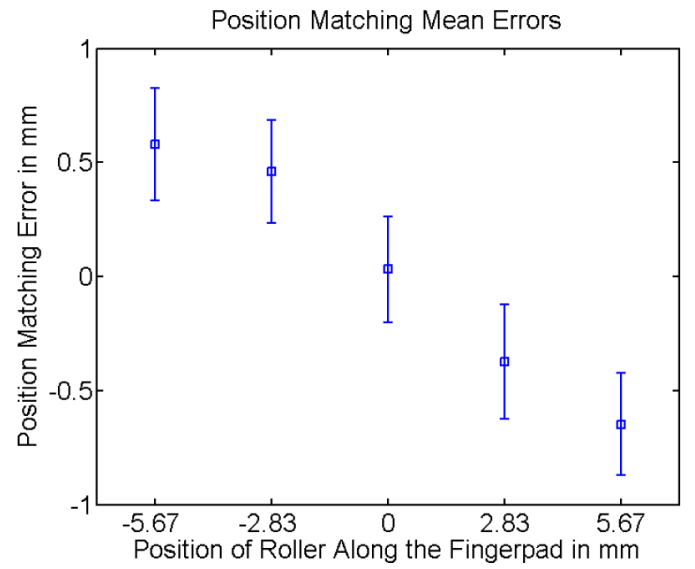

Fig. 8. Mean localization error and its 95 percent confidence intervals among the five test locations across all participants.

virtual model was randomly selected to limit the effect of curvature, proprioceptive, and kinesthetic cues. The cylinder's radius could vary between 50 and $140 \mathrm{~mm}$ (mean $95 \mathrm{~mm}$ ). The fore-aft position of the cylinder was chosen such that users were required to move the CLD's tactile element at least $4 \mathrm{~mm}$ to match its previous position and that a portion of the cylinder would always lie directly below the user's finger. This resulted in the center of the cylinder shifting back and forth by no more than $50 \mathrm{~mm}$ from its starting position over the course of the experiment. The position and radius were also chosen such that the full range of motion of the CLD lays within the workspace of the Phantom force feedback device.

\subsection{Position Matching Results and Discussion}

No effects of testing order or prior experience were observed. The positioning error was evaluated in two ways, the signed error and the absolute error. While positive and negative errors may cancel each other in the average signed error, the absolute error, which is the absolute value of the signed error, is a more stringent measure of the accuracy with which participants could position the tactor.

The mean absolute-error during tactile cue localization for all test locations is approximately $1.3 \mathrm{~mm}$. The mean absolute errors were not found to be statistically different among the five test locations $[\mathrm{F}(4,995)=0.5, \mathrm{p}=0.733]$. This lack of difference indicates that tactile cue localization does not vary with location on the fingerpad. Fig. 7 shows the mean absolute errors and their 95 percent confidence intervals for each of the five test locations across all participants. Hence, to avoid detection of tactile element positioning errors, these errors must be kept less than $1.3 \mathrm{~mm}$. This maximum error is measured for sequential contact of the tactile element. Much smaller tactor motions can be detected when they are experienced instantaneously [28]. Thus, devices require significantly higher position resolution to provide smooth interaction than for detecting position error for sequential contacts. Therefore, even though Doxon et al. [28] suggests that a tactile device will require high-positioning resolution, the above results imply that a device may have significant position error (i.e., $1.3 \mathrm{~mm}$ ) after large motions or sequential contact where the user is more likely to lose their immediate reference. 
The mean localization error, in contrast to the mean absolute error, provides another interesting insight into tactile localization. The mean errors are statistically different with respect to test location $[\mathrm{F}(4,995)=18.92$, $p<0.001$ ] (see Fig. 8). These differences indicate a linear response bias toward the center of the fingerpad. This bias is relatively small compared to the mean absolute error. Fig. 8 shows the mean localization errors and their 95 percent confidence intervals for each of the five test locations across all participants. The error at each test location strongly fits a normal distribution and contains little skew. The normal distribution may also indicate that this bias toward the center of the fingerpad is not likely device related in origin. One possible explanation for this bias is that users naturally orient their fingerpad normal to any surface they are exploring. Doing so positions the CLD's tactor closer to the center of the fingerpad. Participants may have subconsciously adjusted their finger during matching, thus providing a bias toward the center of the fingerpad. Interaction forces with the surface remain relatively constant between trials with forces varying around 1-2 $\mathrm{N}$ depending on the participant. Force levels did not change as a function of position and thus are not a likely cause of this error.

\section{Discrimination OF TACTOR BACKLASH}

The following experiment examines participants' ability to discriminate between the CLD's inherent backlash and an artificially increased backlash. The CLD's inherent backlash is $0.23 \mathrm{~mm}$ and it is not noticeable by the experimenters under typical conditions the CLD system is used. Since the CLD's minimum backlash is nonzero, we treat the experimental task as a discrimination, not a detection, task. However, for all practical purposes, the discrimination thresholds reported here can be viewed as approaching the detection thresholds for backlash under similar conditions.

The discrimination task was performed through a paired comparison (two interval), forced-choice paradigm. Backlash perception is presumably done through a combination of haptic and temporal sensing. Identifying this perceptual limit will allow tactile-kinesthetic devices to potentially include more system backlash, reducing their cost and complexity, while maintaining the imperceptibility of backlash.

Because the effects of backlash are depended on the positioning of the tactile element the threshold was evaluated on low- and high-curvature surfaces as two separate halves of the experiment. This is especially important as the curvature of the surface directly affects the positioning of the tactor for devices utilizing contact location. Fig. 9 shows the finger motion required to produce the same tactor displacement for low- and high-curvature models. On high-curvature models, such as at an edge formed by two faces, the rendered contact location remains stationary on the model (and in the world) as a user moves his/her finger. Thus, the CLD's tactor will move at the same rate as the user's finger in the opposite direction. On lowcurvature models the contact location moves along the surface with the finger, slowing tactor motion with respect to finger motion. This means participants must move their
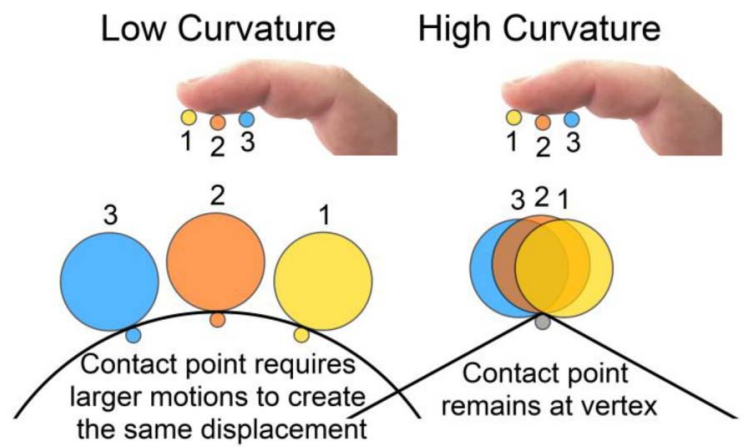

Fig. 9. Contact location positioning on high- and low-curvature surfaces as the finger is moved horizontally left and right. The finger must move farther on low-curvature surfaces to create the same tactor displacement as shown on the high-curvature surface.

finger farther before the tactor is driven enough to overcome the CLD's backlash and begin moving, thus magnifying the deadband created by the backlash and making it easier to detect.

\subsection{Methods}

\subsubsection{Procedure}

This experiment utilized a paired comparison (two interval), forced-choice paradigm, with a 1-up, 2-down adaptive procedure [30]. During each trial the participant was presented with two intervals: a reference interval without added backlash, and a comparison interval with added virtual backlash. The order of the reference and comparison intervals was randomized. Participants were instructed to indicate which of the two intervals contained more backlash. The amount of added backlash increased with each incorrect response and decreased after two consecutive correct responses. The threshold obtained corresponded to the 70.7 percent confidence interval on the psychometric function [30].

Each trial was conducted as follows: First, the participant interacts under the first interval. Once she/he has a feel for the first interval she/he raises the finger and presses "Enter." Two tones sound to let the participant know the second interval is now active. After lowering his/her finger into contact with and interacting with the virtual surface under the second interval the participant raises the finger and presses either " 1 " to indicate the first interval contained more backlash, or " 2 " to indicate the second interval contained more backlash. A single tone sounds, alerting the participant that a new interval 1 is now ready and the next trial is ready to commence.

The experiment continues until the participant has finished 14 reversals. A reversal occurs when the added virtual backlash increases after a decrease, or vice versa. A large step size $(0.3 \mathrm{~mm})$ was used for the first four reversals to provide faster initial convergence. A reduced step size $(0.06 \mathrm{~mm})$ was used for the remaining 10 reversals to provide better accuracy in determining the discrimination threshold. The step sizes for each stage and model were chosen during pilot testing and fixed for all participants.

\subsubsection{Stimuli}

The computed tactor position was augmented with a virtual backlash to emulate larger device backlash levels. Each pair of compared backlash intervals consisted of a 


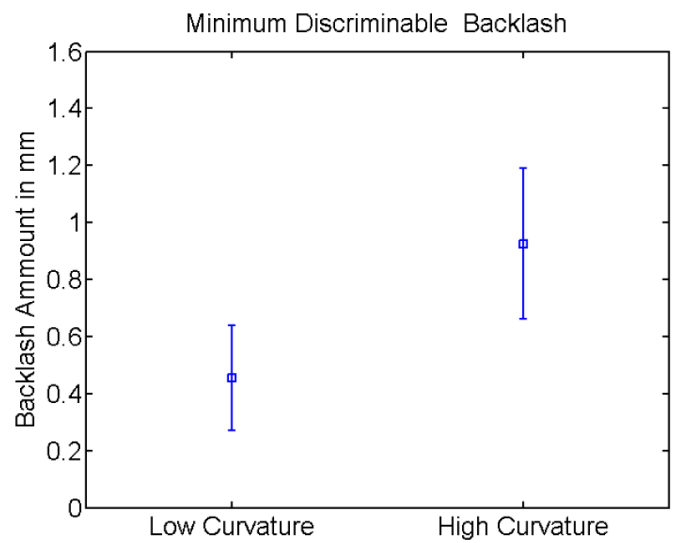

Fig. 10. Minimum discriminable backlash means and their 95 percent confidence intervals for low- and high-curvature models.

virtual model rendered without any added virtual backlash and a model rendered with some small amount of added virtual backlash.

The experiment was split into two halves to evaluate the discriminability of backlash on low- and high-curvature virtual models. In one half of the experiment, participants interacted with the top edge of a horizontally extruded isosceles triangle with a 2 degree angle between its nearly vertical faces. During the other half of the experiment, participants interacted with the base environment's cylinder model ( $95 \mathrm{~mm}$ radius cylinder). In this case, the ratio between the virtual finger radius and the cylinder radius magnifies the effect of the backlash by approximately 7.3:1 (when the participant maintains a horizontal finger orientation). Half of the participants experienced the low curvature model first, while the other half experienced the highcurvature model first.

\subsection{Backlash Results and Discussion}

No effects of testing order or prior experience were observed. The minimum added virtual backlash when tested with both low- and high-curvature virtual models was statistically different from 0 [low curvature: $\mathrm{t}(19)=5.18, \mathrm{p}<0.001$; high curvature: $\mathrm{t}(19)=7.34, \mathrm{p}<0.001]$. After the experiment, most participants reported that their method for detecting backlash involved making a small finger movement and attempting to detect the presence (or the lack) of a corresponding motion of the tactor. This indicates that the haptic portion of the cue is the dominant factor when detecting backlash. This is further supported by the larger positioning errors found in the system delay experiment (Section 7).

The backlash discrimination threshold when interacting with the low-curvature model was approximately $0.46 \mathrm{~mm}$. The backlash discrimination threshold on the high-curvature model was $0.93 \mathrm{~mm}$ (see Fig. 10). As expected, there is a statistically significant decrease in the threshold when interacting with lower curvatures $[\mathrm{F}(1,38)=9.38, \mathrm{p}=$ 0.002] due to the magnified backlash dead band as explained at the beginning of Section 6. Fig. 10 shows the means and 95 percent confidence intervals of the backlash thresholds for both the low and high curvatures. The backlash discrimination threshold is expected to decrease further as curvature decreases. However, at some point the effects of low curvature will slow the tactor motion to an imperceptible degree and backlash can no longer be detected.

As mentioned earlier, the CLD's inherent backlash of $0.23 \mathrm{~mm}$ is not noticeable by the experimenters. Assuming that $0.23 \mathrm{~mm}$ is indeed below the human backlash detection threshold (which requires a no-backlash system to confirm, which is beyond the scope of the present study), our results can also be interpreted as detection thresholds by adding $0.23 \mathrm{~mm}$ to the backlash discrimination thresholds to compute the total system backlash. We would then conclude that the backlash detection thresholds for lowand high-curvature models are approximately 0.69 and $1.16 \mathrm{~mm}$, respectively.

The above backlash perception thresholds were evaluated while participants were specifically looking for backlash. Under general use, participants will not be devoting their full attention to detecting backlash, thus, larger device backlashes on lower curvature models may go unnoticed.

\section{Discrimination of System Delay}

The following experiment examines participants' ability to discriminate between the CLD's inherent system delay and an artificially increased delay. The system delay is defined as the time difference between user action and device reaction. The CLD's inherent delay is around 1-2 ms and not noticeable under typical uses. Strictly speaking, our experiment should be treated as a discrimination task between a nonzero inherent system delay and a delay with additional virtual delay. However, for all practical purposes, the delay discrimination thresholds reported here can be viewed as approaching the delay detection thresholds under similar conditions.

The discrimination task was performed through a paired comparison (two interval), forced-choice paradigm. More system delays will lead to more disassociation between tactile and kinesthetic cues or more sluggish reactions from the system. System delay manifests itself in three forms during a single motion. First, there is a delay in tactor motion after the user's finger has begun moving ("frontend" delay). This delay can be masked by the user's own kinesthetic motion. Second, there is a position error during motion. However, for small system delays this error is too small to be detectable. Lastly, after the user's finger has stopped motion, the tactor will continue its motion for a time ("back-end" delay). This cue, after the participant has stopped moving, is expected to be the most salient as there is no haptic masking of the tactor motion, and the remaining tactor motion can easily be measured temporally.

Perception of the delay was evaluated in two ways to understand the dominant cues in its detection. First, discrimination of system delay was measured as a whole. Second, only the "front-end" component of delay was evaluated.

\subsection{Methods}

\subsubsection{Procedure}

As with the backlash discrimination experiment in Section 6, this experiment utilized a paired comparison (two interval), forced-choice paradigm, with a 1-up, 2-down adaptive procedure [30]. During each trial, the participant was 
presented with two intervals: a reference interval without added delay, and a comparison interval with added virtual system delay. The order of the reference and comparison intervals presented was randomized. Participants were instructed to indicate which of the two intervals contained more system delay. The amount of added delay increased with each incorrect response and decreased after two consecutive correct responses. The threshold obtained corresponded to the 70.7 percent confidence interval on the psychometric function [30].

Each trial was conducted as follows: First, the participant interacts with the first interval. Once they have a feel for that interval they raise their finger and press "Enter." Two tones sound to let the participant know the second interval is now available. After interacting with the second interval, the participant raises his/her finger and presses either " 1 ," to indicate the first surface contained more system delay, or " 2, " to indicate the second surface contained more system delay. A single tone then sounds, alerting the participant that a new interval " 1 " is in place and the next trial can begin.

The experiment continues until the participant has completed 14 reversals. A reversal occurs when the additional virtual system delay increases after a decrease, or vice versa. A large step size (15 ms) was used for the first four reversals to provide faster initial convergence. A reduced step size $(6 \mathrm{~ms})$ was used for the remaining 10 reversals to provide better accuracy in determining the discrimination threshold. The step sizes for each stage and section were chosen during pilot testing and fixed for all participants.

\subsubsection{Stimuli}

Artificial system delay is created by passing the desired tactor position through a FIFO buffer. The length of the FIFO buffer determines the number of haptic cycles the command is delayed. The haptic loop is run at $1 \mathrm{kHz}$ such that each cell in the FIFO buffer delays the signal by $1 \mathrm{~ms}$. As in the backlash discrimination experiment, each set of paired comparisons consists of a model rendered with this additional virtual delay and a model rendered without any virtual delay.

This experiment was split into two halves. Both halves were conducted using the base environment (95- $\mathrm{mm}$ radius cylinder). Pilot testing indicated that curvature had little effect on discrimination of system delay. This is likely due to the fact that the participants could simply move faster to increase the effect of the delay and overcome the slowing effect of low curvature on the tactor motion. During the first half of the experiment, discrimination of whole system delay was evaluated. Participants were allowed to freely interact with the cylinder as desired. During the second half of the experiment, only the "front-end" delay was evaluated. The continued tactor motion after the participant stopped finger movement was removed by restricting user interaction with the model. In this case participants would contact the surface on one side then sweep their finger to the other. When the tactor reached approximately twothirds of the way across its workspace, it would freeze while the participant continued motion, thus eliminating any end of motion cues. The participant would then raise their finger and lower it back onto the surface to unfreeze the tactor and repeat the process.

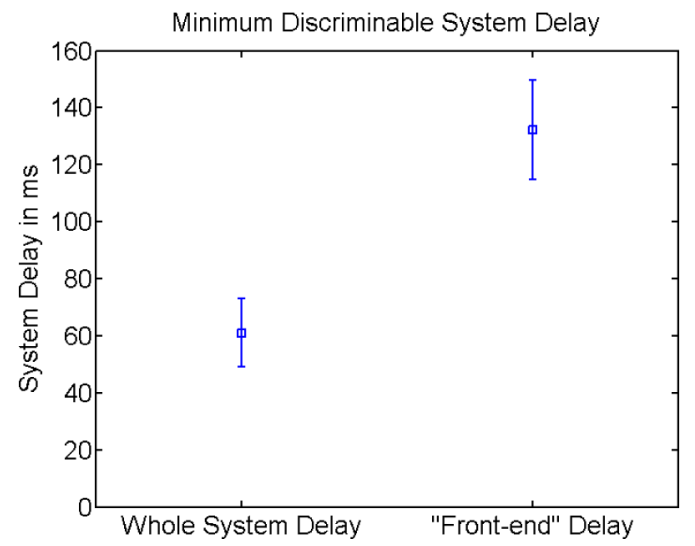

Fig. 11. Mean values for the discrimination thresholds of system delay and their 95 percent confidence intervals for whole system delay and "front-end" delay.

\subsection{Delay Discrimination Results and Discussion}

No effects of testing order or prior experience were observed. The discrimination threshold of system delay was found to be approximately $61 \mathrm{~ms}$. However, when only evaluating the "front-end" delay, the threshold was $132 \mathrm{~ms}$ (see Fig. 11). As expected, the "front-end" delay is significantly less noticeable than the system delay as a whole $[\mathrm{F}(1,38)=49.89$, $\mathrm{p}<0.001]$. This implies that the tactile motion that occurs after the finger has stopped moving is likely the dominant cue when detecting system delay as a whole. The larger threshold of the "front-end" delay can be partially attributed to the restrictions placed on participant's finger motion during that portion of the experiment. However, the majority of the difference can still be attributed to the participant's finger motion masking the delay in tactor motion. Fig. 11 shows the means and 95 percent confidence intervals of the system delay thresholds for both the whole system delay and the "front-end" delay.

The positioning error at the thresholds is larger than the backlash detection threshold. The average position error created by the whole system lag is $0.94 \mathrm{~mm}$. This is about twice the backlash threshold on the low curvature object. Velocities in the "front-end" delay portion were comparable thus resulting in a much larger error. This further supports the argument that the finger motion masks errors in tactor positioning.

The CLD's inherent delay of $1-2 \mathrm{~ms}$ is negligible in comparison to the $61 \mathrm{~ms}$ and $132 \mathrm{~ms}$ discrimination threshold for the whole delay and the "front-end" delay. Assuming that 1-2 ms is below the human delay detection threshold (which requires a no-delay system to confirm, which is beyond the scope of the present study), our discrimination thresholds can also be interpreted as detection thresholds by adding the 1-2 ms to the delay discrimination thresholds. Numerically, it does not make much difference whether the $1-2 \mathrm{~ms}$ is added to the discrimination thresholds to obtain the corresponding detection thresholds for the whole and "front-end" delays.

The system delay measured here represents the settling time of the system as a whole and can be used to improve devices in a variety of ways. This delay can take the form of larger device inertia or a slower settling controller with lower gains for improved stability. As with the backlash 


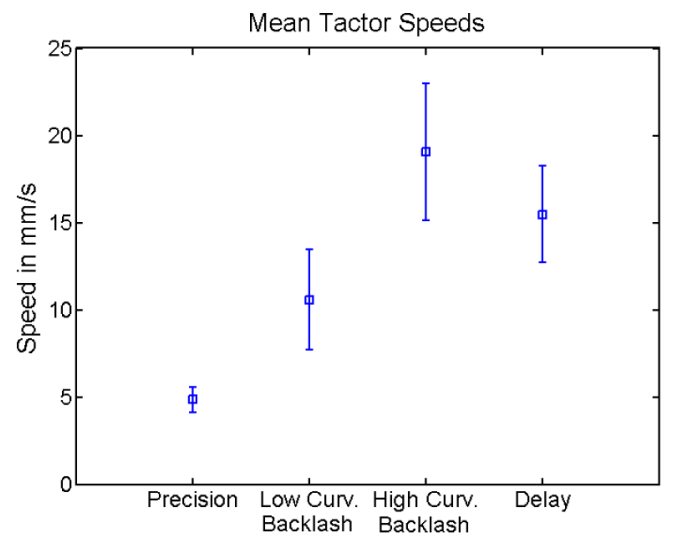

Fig. 12. Tactor velocity mean and 95 percent confidence intervals during each experimental condition. Front delay not evaluated as participant motions were restricted.

perception experiment in Section 6, participants will not be actively looking for system delay during most uses, thus larger system delays may go unnoticed.

\section{Velocity Data}

During each of the three presented experiments the position and velocity of the participant's finger and the device's tactor was captured. This data provide valuable insights into common interaction speeds when exploring simple virtual environments. Velocities from the "front-end" delay experimental task are not included in this analysis as participant motion was restricted and does not represent natural participant interaction. These velocities were similar in magnitude to those in the unrestricted whole system delay case.

Average finger velocity during exploration of lowcurvature surfaces varied between $32 \mathrm{~mm} / \mathrm{s}$ for precision motions to $74 \mathrm{~mm} / \mathrm{s}$ during fast motions. Tactor velocities are significantly lower than finger velocities in the majority of the experiment due to the slowing effect of low-curvature surfaces. Tactor velocities ranged between $5 \mathrm{~mm} / \mathrm{s}$ for precision tasks and $19 \mathrm{~mm} / \mathrm{s}$ during the high-curvature backlash task where tactor speed was equal to finger speeds (when a single finger orientation is maintained). The reported velocities on low-curvature objects indicate a ratio between tactor and finger velocity closer to $5: 1$ as opposed to the expected 7.3:1 ratio between the finger model and object model. However, the collected position and orientation data shows participants rolling their fingers as they explored the low-curvature surfaces. The 7.3:1 ratio is only true if no orientation changes occur during motion. Figs. 12 and 13 show the mean and 95 percent confidence intervals of recorded tactor and finger velocities for all participants under each experimental condition.

These recorded velocities provide insight into the necessary responsiveness of tactile devices. Such devices should be capable of tactor motions in excess of $20 \mathrm{~mm} / \mathrm{s}$, though the majority of tactile exploration on low-curvature models appears to occur below $10 \mathrm{~mm} / \mathrm{s}$. Ideally, tactile devices should be capable of velocities exceeding maximum finger exploration (near $70 \mathrm{~mm} / \mathrm{s}$ ) as tactor velocities match finger velocities on high-curvature surfaces. While finger

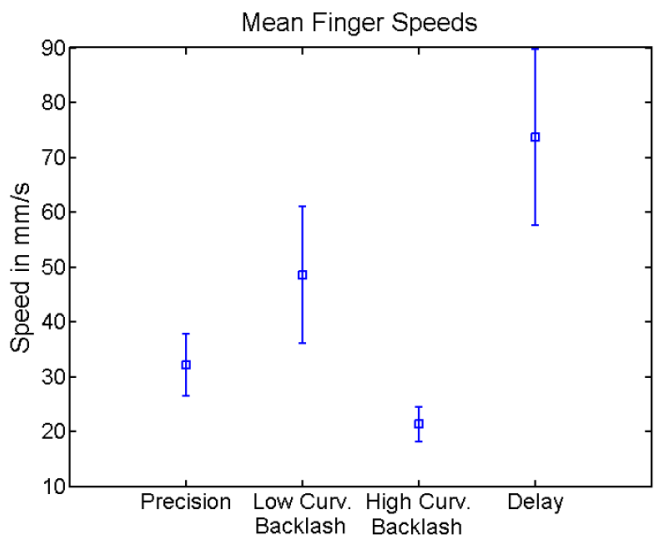

Fig. 13. Finger velocity mean and 95 percent confidence intervals during each experimental condition. Front delay not evaluated as participant motions were restricted.

and desired tactor velocities can exceed $200 \mathrm{~mm} / \mathrm{s}$, it is unlikely that users will be able to actively discern surface features at those speeds, thus making this high velocity an unnecessary design requirement.

\section{SUmmary AND Future Work}

Three experiments were run to evaluate factors relevant to tactile display device design. The first of these experiments identifies the resolution with which the user is able to repeatedly place a contact at a given location on the fingerpad. Participants are able to localize tactile cues to within $1.3 \mathrm{~mm}$ on their fingerpad. Cue localization is biased toward the center of the fingerpad. These results stipulate the maximum positioning error the device should achieve after large or sequential motion.

The second experiment evaluates the minimum perceivable difference in backlash in positioning a tactile element. Subjects were able to discriminate device backlash in excess of $0.46 \mathrm{~mm}$ on low-curvature models and $0.93 \mathrm{~mm}$ on highcurvature models. Since the device's inherent backlash $(0.23 \mathrm{~mm})$ is most likely below human detection threshold, the discrimination results are interpreted as backlash detection thresholds when the device's inherent backlash is taken into account. Accordingly, backlash becomes detectable at levels as low as $0.69 \mathrm{~mm}$ on low-curvature models. High-curvature models make backlash detection more difficult, increasing the threshold to $1.16 \mathrm{~mm}$. The haptic portion of backlash was found to be the dominant cue used in detection. In contrast to the first experiment these thresholds indicate the positioning requirements for small or immediate motions.

The third experiment measures the minimum perceivable difference in system delay between user action and device motion. Since the CLD's inherent delay (1-2 ms) is negligible, the discrimination results can be interpreted as delay detection thresholds. Therefore, system delay on tactile output can be as large as $61 \mathrm{~ms}$ before it can be detected. The back-end delay (tactile motion after user motion has ceased) was the dominant cue of system delay. Front-end delay is masked by finger motion and was found to become detectable at around $132 \mathrm{~ms}$. The position error at the thresholds was found to be larger than the detection 
threshold for backlash on the same model, further indicating the masking effects of motion and the dominance of the haptic portion of backlash cues when detecting the threshold. These results determine the allowable system delay before it is noticeable.

During each experiment, the velocities of both the tactor and finger were recorded. Subjects explored low-curvature models with finger velocities ranging from $32 \mathrm{~mm} / \mathrm{s}$ for precision motions to $74 \mathrm{~mm} / \mathrm{s}$ during fast motions. Tactor velocities are significantly lower than finger velocities in the majority of the experiment due to the slowing effect of lowcurvature surfaces (see Section 6). As such, devices should be capable of tactor velocities in excess of $20 \mathrm{~mm} / \mathrm{s}$, but ideally be able to exceed the $74 \mathrm{~mm} / \mathrm{s}$ finger velocity found during rapid exploration.

The above evaluated perceptual limits provide the foundation needed to design smaller, less expensive, and more capable tactile devices, expanding their presence as both research and commercial products, while creating perceptibly identical devices.

Future work will involve designing a more compact 2 degrees-of-freedom contact location display based on the above guidelines. Use of this new device is aimed at providing more insight into tactile interaction in multifinger manipulation.

\section{ACKNOWLEDGMENTS}

This work was supported, in part, by the US National Science Foundation under awards IIS-0904456 and IIS-0904423.

\section{REFERENCES}

[1] S. Biggs and M. Srinivasan, "Haptic Interfaces," Handbook of Virtual Environments: Design, Implementation, and Applications, pp. 93-116, Lawrence Erlbaum Assoc., 2002.

[2] K. Hale and K. Stanney, "Deriving Haptic Design Guidelines from Human Physiological, Psychophysical, and Neurological Foundations," Proc. IEEE Conf. Computer Graphics and Applications, vol. 24, no. 2, pp. 33-39, 2004.

[3] B. Gleeson, S. Horschel, and W. Provancher, "Perception of Direction for Applied Tangential Skin Displacement: Effects of Speed, Displacement and Repetition," IEEE Trans. Haptics, Special Section on the World Haptics Conf., vol. 3, no. 3, pp. 177188, July-Sept. 2010.

[4] J. Loomis and C. Collins, "Sensitivity to Shifts of a Point Stimulus: An Instance of Tactile Hyperacuity," Attention, Perception, $\mathcal{E}$ Psychophysics, vol. 24, no. 6, pp. 487-492, 1978.

[5] J. Loomis, "An Investigation of Tactile Hyperacuity," Sensory Processes, vol. 3, pp. 289-302, 1979.

[6] R. Boven and K. Johnson, "The Limit of Tactile Spatial Resolution in Humans: Grating Orientation Discrimination at the Lip, Tongue, and Finger," Neurology, vol. 44, no. 12, pp. 2361-2361, 1994.

[7] T.B. Sheridan and W.R. Ferrell, "Remote Manipulative Control with Transmission Delay," IEEE Trans. Human Factors in Electronics, vol. HFE-4, no. 1, pp. 25-29, Sept. 1963.

[8] B. Adelstein, D. Begault, M. Anderson, and E. Wenzel, "Sensitivity to Haptic-Audio Asynchrony," Proc. ACM Fifth Int'l Conf. Multimodal Interfaces, 2003.

[9] K. Mania, B. Adelstein, S. Ellis, and M. Hill, "Perceptual Sensitivity to Head Tracking Latency in Virtual Environments with Varying Degrees of Scene Complexity," Proc. ACM First Symp. Applied Perception in Graphics and Visualization, 2004.

[10] C. Jay and R. Hubbold, "Delayed Visual and Haptic Feedback in a Reciprocal Tapping Task," Proc. First Joint Eurohaptics Conf. and Symp. Haptic Interfaces for Virtual Environment and Teleoperator Systems (WHC '05), 2005.

[11] T. Brooks, "Telerobotic Response Requirements," Proc. IEEE Int'l Conf. Systems, Man and Cybernetics, 1990.
[12] A. Frisoli, M. Bergamasco, S. Wu, and E. Ruffaldi, "Evaluation of Multipoint Contact Interfaces in Haptic Perception of Shapes," Multi-Point Interaction with Real and Virtual Objects, Springer Tracts in Advanced Robotics, vol. 18, pp. 177-188, 2005.

[13] S. Lederman and R. Klatzky, "Hand Movements: A Window into Haptic Object Recognition," Cognitive Psychology, vol. 19, no. 3, pp. 342-368, 1987.

[14] S. Lederman and R. Klatzky, "Extracting Object Properties through Haptic Exploration," Acta Psychologica, vol. 84, no. 1, pp. 29-40, 1993.

[15] R.J. Webster III, T.E. Murphy, L.N. Verner, and A.M. Okamura, "A Novel Two-Dimensional Tactile Slip Display: Design, Kinematics and Perceptual Experiments," ACM Trans. Applied Perception, vol. 2, no. 2, pp. 150-165, 2005.

[16] K. Drewing, M. Fritschi, R. Zopf, M.O. Ernst, and M. Buss, "First Evaluation of a Novel Tactile Display Exerting Shear Force via Lateral Displacement," ACM Trans. Applied Perception, vol. 2, no. 2, pp. 118-131, 2005.

[17] C. Salisbury, B. Gillespie, H. Tan, F. Barbagli, and K. Salisbury, "What You Can't Feel Won't Hurt You: Evaluating Haptic Hardware Using a Haptic Sensitivity Contrast Function," IEEE Trans. Haptics, vol. 4, no. 2, pp. 134-146, Apr.-June 2011.

[18] L.A. Jones and N.B. Sarter, "Tactile Displays: Guidance for Their Design and Application," J. Human Factors and Ergonomics Soc., vol. 50, no. 1, pp. 90-111, 2008.

[19] K.S. Hale and K.M. Stanney, "Deriving Haptic Design Guidelines from Human Physiological, Psychophysical, and Neurological Foundations," Computer Graphics and Applications, IEEE, vol. 24, no. 2, pp. 33-39, Mar. 2004.

[20] G. Moy, U. Singh, E. Tan, and R.S. Fearing, "Human Psychophysics for Teletaction System Design," Haptics-e, vol. 1, no. 3, pp. 120, 2000.

[21] M. Salada, J. Colgate, P. Vishton, and E. Frankel, "An Experiment on Tracking Surface Features with the Sensation of Slip," Proc. First Joint EuroHaptics Conf. and Symp. Haptic Interfaces for Virtual Environment and Teleoperator Systems (WHC '05), pp. 132-137, 2005.

[22] M. Fritschi, M. Ernst, and M. Buss, "Integration of Kinesthetic and Tactile Display - A Modular Design Concept," EuroHaptics Conf., 2006.

[23] I. Sarakoglou, N. Garcia-Hernandez, N. Tsagarakis, and D. Caldwell, "A High Performance Tactile Feedback Display and Its Integration in Teleoperation," IEEE Trans. Haptics, vol. 5, no. 3, pp. 252-263, Jan.-Mar. 2012.

[24] A. Frisoli, M. Solazzi, F. Salsedo, and M. Bergamasco, "A Fingertip Haptic Display for Improving Curvature Discrimination," Presence: Teleoperators and Virtual Environments, vol. 17, no. 6, pp. 550561, Oct. 2008

[25] H. Dostmohamed and V. Hayward, "Trajectory of Contact Region on the Fingerpad Gives the Illusion of Haptic Shape," Experimental Brain Research, vol. 164, no. 3, pp. 387-394, July, 2005.

[26] F. Chinello, M. Malvezzi, C. Pacchierotti, and D. Prattichizzo, "A Three DoFs Wearable Tactile Display for Exploration and Manipulation of Virtual Objects," Proc. IEEE, Haptics Symp. (HAPTICS '12), pp. 71-76, 2012.

[27] W.R. Provancher, M.R. Cutkosky, K.J. Kuchenbecker, and G. Niemeyer, "Contact Location Display for Haptic Perception of Curvature and Object Motion," Int'l J. Robotics Research, vol. 24, no. 9, pp. 691-702, 2005.

[28] A.J. Doxon, D.E. Johnson, H.Z. Tan, and W.R. Provancher, "Force and Contact Location Shading Methods for Use Within Two-and Three-Dimensional Polygonal Environments," Presence: Teleoperators and Virtual Environments, vol. 20, no. 6, pp. 505-528, 2011.

[29] N.I. Durlach, L.A. Delhorne, A. Wong, W.Y. Ko, W.M. Rabinowitz and J. Hollerbach, "Manual Discrimination and Identification of Length by the Finger-Span Method," Perception $\mathcal{E}$ Psychophysics, vol. 46 , no. 1 , pp. 29-38, 1989.

[30] H. Levitt, "Transformed Up-Down Methods in Psychoacoustics," J. Acoustical Soc. of Am., vol. 49, pp. 467-477, 1971. 


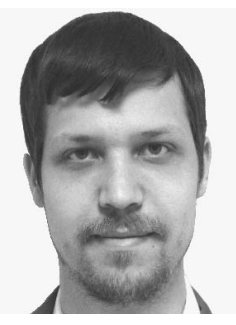

Andrew J. Doxon received the BS degree in electrical engineering from the New Mexico Institute of Mining and Technology in 2008 and the MS degree in electrical and computer engineering from the University of Utah in 2010. He is working toward the PhD degree in mechanical engineering at the University of Utah. His primary research interests are on improving combined tactile and kinesthetic haptic devices, by improving the design of new tactile devices, and the algorithms that drive them. He is a student member of the IEEE.

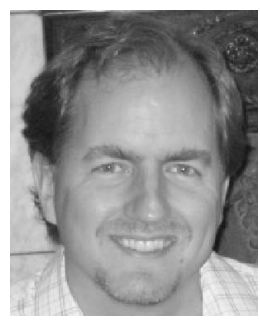

David E. Johnson received the BA degree in computer science and physics from Carleton College and the $\mathrm{PhD}$ degree in computer science from the University of Utah, focusing on geometric computations for haptic rendering. $\mathrm{He}$ is a research scientist at the University of Utah's School of Computing. His current interests are in applying geometric computations to the area of robotics.

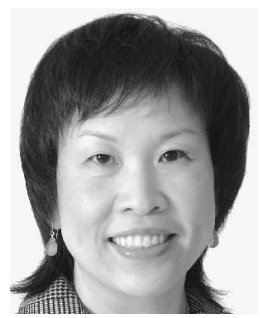

Hong Z. Tan received the bachelor's degree in biomedical engineering in 1986 from Shanghai Jiao Tong University and the master's and doctorate degrees both in electrical engineering and computer science from the Massachusetts Institute of Technology (MIT), in 1988 and 1996, respectively. She was a research scientist at the MIT Media Lab from 1996 to 1998 before joining the faculty at Purdue University. She is currently a professor of electrical and computer engineering, with courtesy appointments in the school of mechanical engineering and the department of psychological sciences. She founded and directs the Haptic Interface Research Laboratory at Purdue University. She is currently editor-in-chief of the World Haptics Conference editorial board. She served as the founding chair of the IEEE Technical Committee on Haptics from 2006 to 2008. She was a recipient of the US National Science Foundation CAREER Award from 2000 to 2004. Her research interest are on haptic human-machine interfaces in the areas of haptic perception, rendering and multimodal performance. She is a senior member of the IEEE.

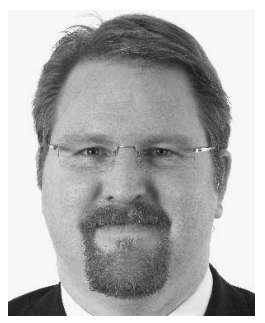

William R. Provancher received the BS degree in mechanical engineering and the MS degree in materials science and engineering, both from the University of Michigan, and the $\mathrm{PhD}$ degree from the Department of Mechanical Engineering, Stanford University. His PhD was in the area of haptics, tactile sensing, and feedback. His postdoctoral research involved the design of bioinspired climbing robots. He is currently a tenured associate professor in the Department of Mechanical Engineering, University of Utah. He teaches courses in the areas of mechanical design, mechatronics, and haptics. His active areas of research include haptics and tactile feedback. He is an associate editor of the IEEE Transactions on Haptics and cochair of the Technical Committee on Haptics. He received an US National Science Foundation CAREER Award in 2008 and won Best Paper and Poster Awards at the 2009 and 2011 World Haptics Conferences for his work on tactile feedback. He is a member of the IEEE.

For more information on this or any other computing topic, please visit our Digital Library at www.computer.org/publications/dlib. 